International Journal of Scientific Research in

\title{
Hamiltonian Decomposition of Special Class of Ladder Graphs
}

\author{
L.T. Cherin Monish Femila ${ }^{1^{*}}$ and S. Asha ${ }^{2}$ \\ ${ }^{1}$ Dept. of Mathematics, Nesamony Memorial Christian College, Marthandam, INDIA \\ ${ }^{2}$ Dept. of Mathematics, Nesamony Memorial Christian College, Marthandam, INDIA \\ (Affiliated to Manonmaniam Sundaranar University, Abishekapatti, INDIA) \\ "Corresponding Author: cherinmonishfemila@ gmail.com, Research Scholar (Reg.No.12604)
}

Available online at: www.isroset.org

Received: 04/Oct/2018, Accepted: 19/Oct/2018, Online: 31/Oct/2018

\begin{abstract}
A path in a graph G that contains every vertex of G is called Hamiltonian path. A cycle in a graph G that contains every vertex of $\mathrm{G}$ is called a Hamiltonian cycle of G. A Hamiltonian graph is a graph that contains a Hamiltonian cycle. A graph is uniquely Hamiltonian if it contains exactly one Hamiltonian cycle. A decomposition of a graph $\mathrm{G}$ into Hamiltonian cycles will be called Hamiltonian decomposition of G. In this paper, we decompose some families of graphs such as ladder, triangular ladder, diagonal ladder, and circular ladder using the concept of Hamiltonian decomposition. Also using the concept of Hamiltonian decomposition of ladder graphs we decompose the diagonal ladder graph.
\end{abstract}

Keywords - Hamiltonian cycle, Hamiltonian graph, uniquely Hamiltonian, Hamiltonian decomposition, Ladder Graph

\section{INTRODUCTION}

Graph theory is proved to be tremendously useful in modeling the essential features of systems with finite components. Graphical models are used to represent telephone network, railway network, communication problems, traffic network etc. A graph is a convenient way of representing information involving relationship between objects. The objects are represented by vertices and the relations by edges. The concept of decomposition of graphs in Hamiltonian cycles, Hamiltonian path decomposition of regular graphs was introduced by Klas Markstrom[1]. Decomposition of complete graphs into Hamilton cycles was discussed in [2] and J.C. Bermond [3] deals about Hamiltonian Decompositions of Graphs, Directed Graphs and Hypergraphs.

A path in a graph $G$ that contains every vertex of $G$ is called Hamiltonian path. A cycle in a graph $G$ that contains every vertex of $G$ is called a Hamiltonian cycle of G. A Hamiltonian graph is a graph that contains a Hamiltonian cycle. A graph is uniquely Hamiltonian if it contains exactly one Hamiltonian cycle. A decomposition of a graph G into Hamiltonian cycles will be called Hamiltonian decomposition of $\mathrm{G}$.

The Ladder graph $L_{n}, n \geq 2$ is defined by $P_{n} \times P_{2}$, where $P_{n}$ is a path with $n$ vertices and $x$ denotes the Cartesian product and $P_{2}$ is a path with two vertices.

A Triangular Ladder graph $\mathbf{T L}_{\mathbf{n}}, \mathrm{n} \geq 2$ is a graph obtained from the Ladder graph $L_{n}=P_{n} \times P_{2}$ by adding the edges $u_{i} v_{i+1}$ for $1 \leq \mathrm{i} \leq \mathrm{n}-1$.

The Diagonal Ladder graph is a Ladder graph with additional edges $u_{i} v_{i+1}$ and $v_{i} u_{i+1}$, denoted by $\mathbf{D L} \mathbf{L}_{\mathbf{n}}$.

The Circular Ladder graph $\mathbf{C L}_{\mathbf{n}}, \mathrm{n} \geq 3$ is defined by $\mathrm{CL}_{\mathrm{n}}=\mathrm{C}_{\mathrm{n}} \times \mathrm{K}_{2}$, where $\mathrm{C}_{\mathrm{n}}$ is a cycle with $\mathrm{n}$ vertices and $\mathrm{x}$ denotes the cartesian product and $\mathrm{K}_{2}$ is a complete graph with two vertices.

Section I contains the introduction of Hamiltonian decomposition of graphs and related topics, Section II contains Hamiltonian decomposition of ladder graphs, Section III contains Hamiltonian decomposition of triangular ladder graphs, Section IV contains Hamiltonian decomposition of diagonal ladder graphs, Section V contains Hamiltonian decomposition of circular ladder graphs , Section VI concludes research work. 


\section{HAMILTONIAN DECOMPOSITION OF LADDER GRAPH $\mathrm{L}_{\mathbf{n}}$}

Theorem: 2.1

The Ladder graph $L_{n}, n \geq 2$ is uniquely Hamiltonian and the rest of the edges form $n-2$ copies of $K_{2}$.

Proof:

Let $\mathrm{L}_{\mathrm{n}}, \mathrm{n} \geq 2$ be the Ladder graph with vertex set $\mathrm{V}:\left\{\mathrm{u}_{1}, \mathrm{u}_{2}, \mathrm{u}_{3}, \ldots, \mathrm{u}_{\mathrm{n}}, \mathrm{v}_{1}, \mathrm{v}_{2}, \mathrm{v}_{3}, \ldots, \mathrm{v}_{\mathrm{n}}\right\}$. By definition, clearly we get two paths $\mathrm{Q}_{1}$ and $\mathrm{Q}_{2}$ of length $n, Q_{1}: u_{1} u_{2} u_{3} \ldots u_{n}$ and $Q_{2}: v_{1} v_{2} v_{3} \ldots v_{n}$.

Form a spanning cycle: connect the vertex $u_{1}$ of $Q_{1}$ to $v_{1}$ of $Q_{2}$ and connect the vertex $u_{n}$ of $Q_{1}$ to $v_{n}$ of $Q_{2}$ at the same time. This is the only Hamiltonian cycle. Because connecting any vertex $\mathrm{u}_{\mathrm{i}}, 2 \leq \mathrm{i} \leq \mathrm{n}-1$ of $\mathrm{Q}_{1}$ to $\mathrm{v}_{\mathrm{i}}, 2 \leq \mathrm{i} \leq \mathrm{n}-1$ of $\mathrm{Q}_{2}$ makes a cycle which is not a spanning cycle. Therefore, $L_{n}, n \geq 2$ is uniquely Hamiltonian. The remaining edges joining the vertices $u_{i}, 2 \leq i \leq n-1$ of $Q_{1}$ to $v_{i}, 2 \leq i \leq n-1$ of $Q_{2}$ makes $n-2$ copies of $\mathrm{K}_{2}$.

\section{Example:2.2}

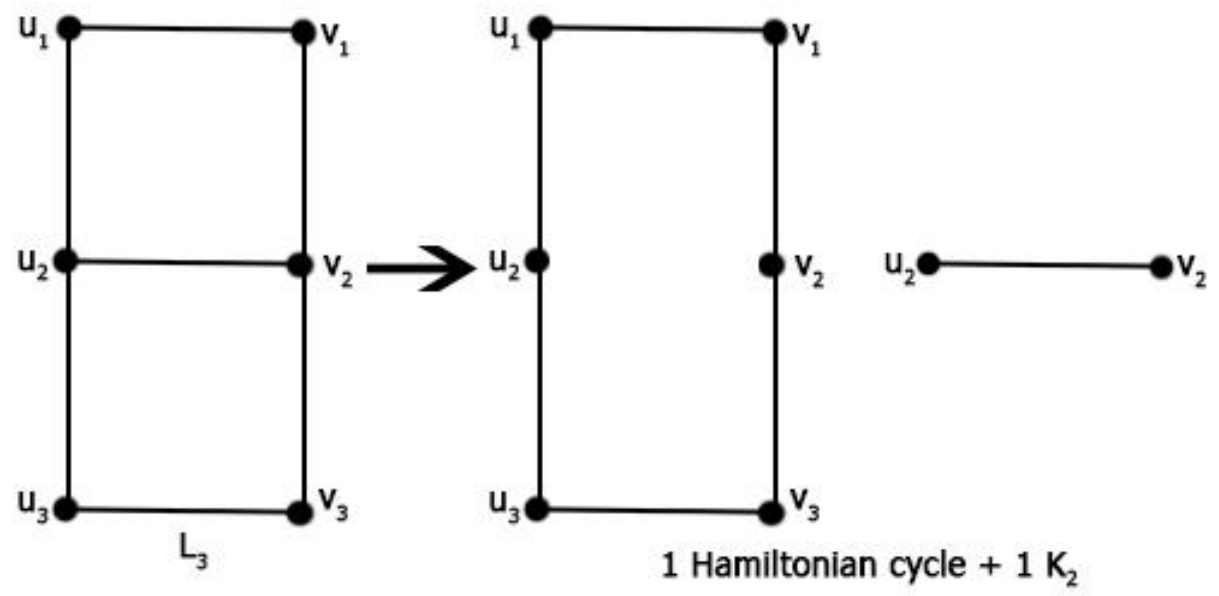

\section{HAMILTONIAN DECOMPOSITION OF TRIANGULAR LADDER GRAPH TL}

\section{Theorem: 3.1}

The Triangular Ladder graph $\mathrm{TL}_{\mathrm{n}}, \mathrm{n} \geq 2$ is uniquely Hamiltonian and the rest of the edges form a path $\mathrm{P}_{2 \mathrm{n}-2}$.

Proof:

Let $\mathrm{TL}_{\mathrm{n}}, \mathrm{n} \geq 2$ be the Triangular Ladder graph with vertex set $\mathrm{V}:\left\{\mathrm{u}_{1}, \mathrm{u}_{2}, \mathrm{u}_{3}, \ldots, \mathrm{u}_{\mathrm{n}}, \mathrm{v}_{1}, \mathrm{v}_{2}, \mathrm{v}_{3}, \ldots, \mathrm{v}_{\mathrm{n}}\right\}$. By definition, clearly we get two paths $\mathrm{Q}_{1}$ and $\mathrm{Q}_{2}$ of length $\mathrm{n}, \mathrm{Q}_{1}: \mathrm{u}_{1} \mathrm{u}_{2} \mathrm{u}_{3} \ldots \mathrm{u}_{\mathrm{n}}$ and $\mathrm{Q}_{2}: \mathrm{v}_{1} \mathrm{v}_{2} \mathrm{v}_{3} \ldots \mathrm{v}_{\mathrm{n}}$.

Form a spanning cycle: connect the vertex $u_{1}$ of $Q_{1}$ to $v_{1}$ of $Q_{2}$ and connect the vertex $u_{n}$ of $Q_{1}$ to $v_{n}$ of $Q_{2}$ at the same time. This is the only Hamiltonian cycle. Because connecting any vertex $\mathrm{u}_{\mathrm{i}}, 2 \leq \mathrm{i} \leq \mathrm{n}-1$ of $\mathrm{Q}_{1}$ to $\mathrm{v}_{\mathrm{i}}, 2 \leq \mathrm{i} \leq \mathrm{n}-1$ of $\mathrm{Q}_{2}$ or connecting any vertex $\mathrm{u}_{\mathrm{i}}, 1 \leq \mathrm{i} \leq \mathrm{n}-1$ of $\mathrm{Q}_{1}$ to $\mathrm{v}_{\mathrm{i}}, 2 \leq \mathrm{i} \leq \mathrm{n}$ of $\mathrm{Q}_{2}$ makes a cycle which is not a spanning cycle. Therefore, $\mathrm{TL}_{\mathrm{n}}, \mathrm{n} \geq 2$ is uniquely Hamiltonian. The remaining edges joining the vertices $\mathrm{u}_{\mathrm{i}}, 2 \leq \mathrm{i} \leq \mathrm{n}-1$ of $\mathrm{Q}_{1}$ to $\mathrm{v}_{\mathrm{i}}, 2 \leq \mathrm{i} \leq \mathrm{n}-1$ of $\mathrm{Q}_{2}$ or connecting any vertex $\mathrm{u}_{\mathrm{i}}, 1 \leq \mathrm{i} \leq \mathrm{n}-1$ of $\mathrm{Q}_{1}$ to $\mathrm{v}_{\mathrm{i}}, 2 \leq \mathrm{i} \leq \mathrm{n}$ of $\mathrm{Q}_{2}$ makes a path $\mathrm{P}_{2 \mathrm{n}-2}$. 


\section{Example: 3.2}
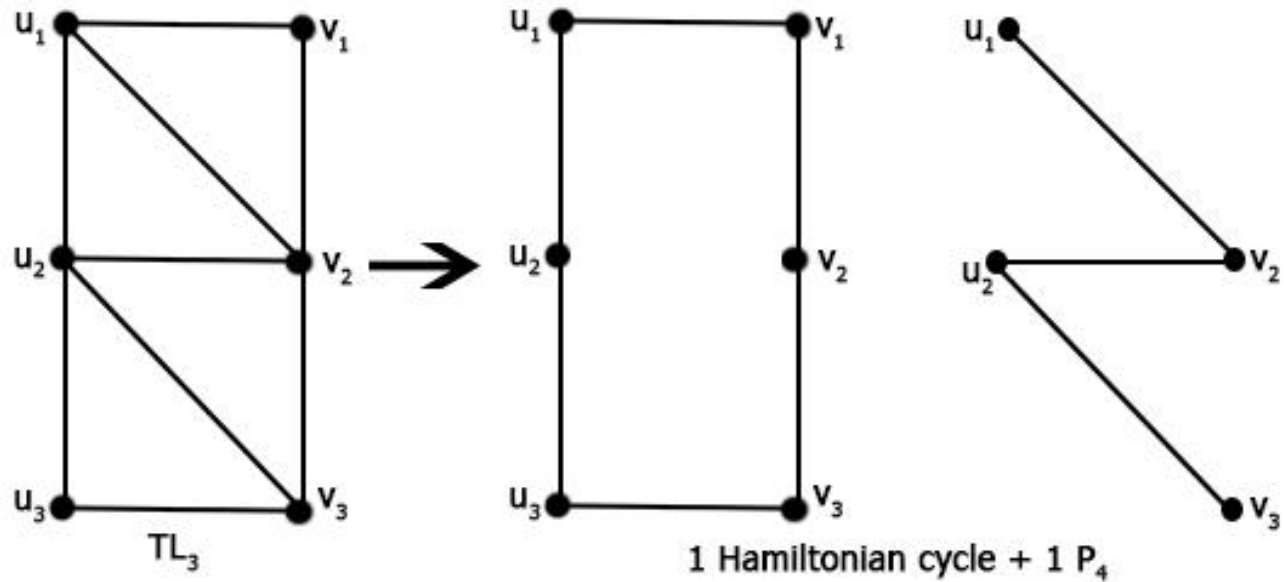

\section{HAMILTONIAN DECOMPOSITION OF DIAGONAL LADDER GRAPH DL}

\section{Theorem: 4.1}

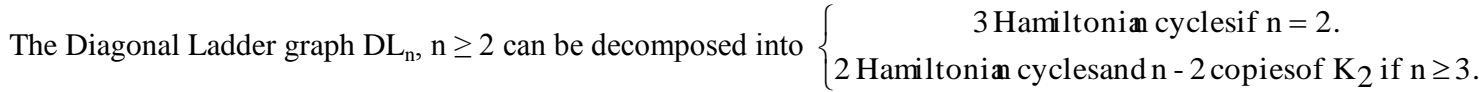

Proof:

Let $\mathrm{DL}_{\mathrm{n}}, \mathrm{n} \geq 2$ be the Diagonal Ladder graph with vertex set $\mathrm{V}:\left\{\mathrm{u}_{1}, \mathrm{u}_{2}, \mathrm{u}_{3}, \ldots, \mathrm{u}_{\mathrm{n}}, \mathrm{v}_{1}, \mathrm{v}_{2}, \mathrm{v}_{3}, \ldots, \mathrm{v}_{\mathrm{n}}\right\}$.

Case 1: $\mathrm{n}=2$

Let $\mathrm{DL}_{2}$ be the Diagonal Ladder graph with vertex set $\mathrm{V}:\left\{\mathrm{u}_{1}, \mathrm{u}_{2}, \mathrm{v}_{1}, \mathrm{v}_{2}\right\}$. By definition, $\mathrm{DL}_{2}$ is obtained from $\mathrm{L}_{2}$ which is uniquely Hamiltonian, so that $\mathrm{DL}_{2}$ has a Hamiltonian cycle. Also $\mathrm{DL}_{2}$ has additional edges $\mathrm{u}_{1} \mathrm{v}_{2}$ and $\mathrm{u}_{2} \mathrm{v}_{1}$. Connecting these two edges in two ways, we get two Hamiltonian cycles. Therefore, $\mathrm{DL}_{2}$ has 3 Hamiltonian cycles.

Case 2: $n \geq 3$

By definition, $\mathrm{DL}_{\mathrm{n}}$ is obtained from $\mathrm{L}_{\mathrm{n}}$ which is uniquely Hamiltonian, so that $\mathrm{DL}_{\mathrm{n}}$ has a Hamiltonian cycle. Now, we construct two paths $\mathrm{Q}_{1}$ and $\mathrm{Q}_{2}$ with the additional edges $\mathrm{u}_{\mathrm{i}} \mathrm{v}_{\mathrm{i}+1}$ and $\mathrm{u}_{\mathrm{i}+1} \mathrm{v}_{\mathrm{i}}$ of length $\mathrm{n}, \mathrm{Q}_{1}: \mathrm{u}_{1} \mathrm{v}_{2} \mathrm{u}_{3} \mathrm{v}_{4} \ldots \mathrm{u}_{\mathrm{n}-3} \mathrm{v}_{\mathrm{n}-2} \mathrm{u}_{\mathrm{n}-1} \mathrm{v}_{\mathrm{n}}$ and $\mathrm{Q}_{2}: \mathrm{v}_{1} \mathrm{u}_{2} \mathrm{v}_{3} \mathrm{u}_{4} \ldots \mathrm{v}_{\mathrm{n}-3} \mathrm{u}_{\mathrm{n}-2} \mathrm{v}_{\mathrm{n}-1} \mathrm{u}_{\mathrm{n}}$. Form a spanning cycle: connect the vertex $\mathrm{u}_{1}$ of $\mathrm{Q}_{1}$ to $\mathrm{v}_{1}$ of $\mathrm{Q}_{2}$ and connect the vertex $\mathrm{v}_{\mathrm{n}}$ of $\mathrm{Q}_{1}$ to $\mathrm{u}_{\mathrm{n}}$ of $\mathrm{Q}_{2}$ at the same time. Therefore, $\mathrm{DL} \mathrm{n}_{\mathrm{n}}$, $\mathrm{n} \geq 3$ contains 2 Hamiltonian Cycles. Also the remaining edges joining the vertices $\mathrm{u}_{\mathrm{i}}, 2 \leq \mathrm{i} \leq \mathrm{n}-1$ of $\mathrm{Q}_{1}$ to $\mathrm{v}_{\mathrm{i}}, 2 \leq \mathrm{i} \leq \mathrm{n}-1$ of $\mathrm{Q}_{2}$ makes $\mathrm{n}-2$ copies of $\mathrm{K}_{2}$.

\section{Example: 4.2}
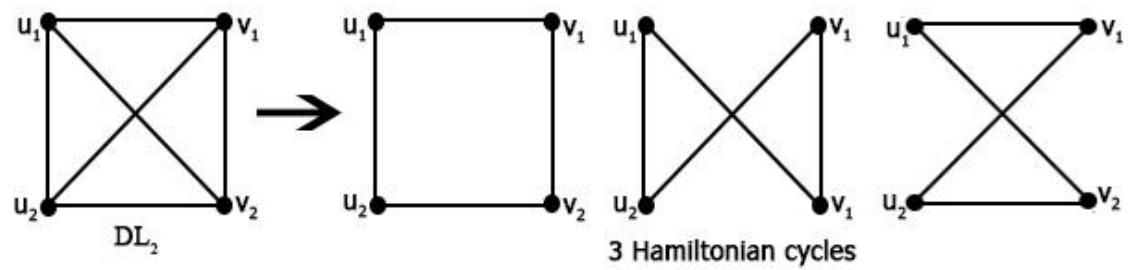


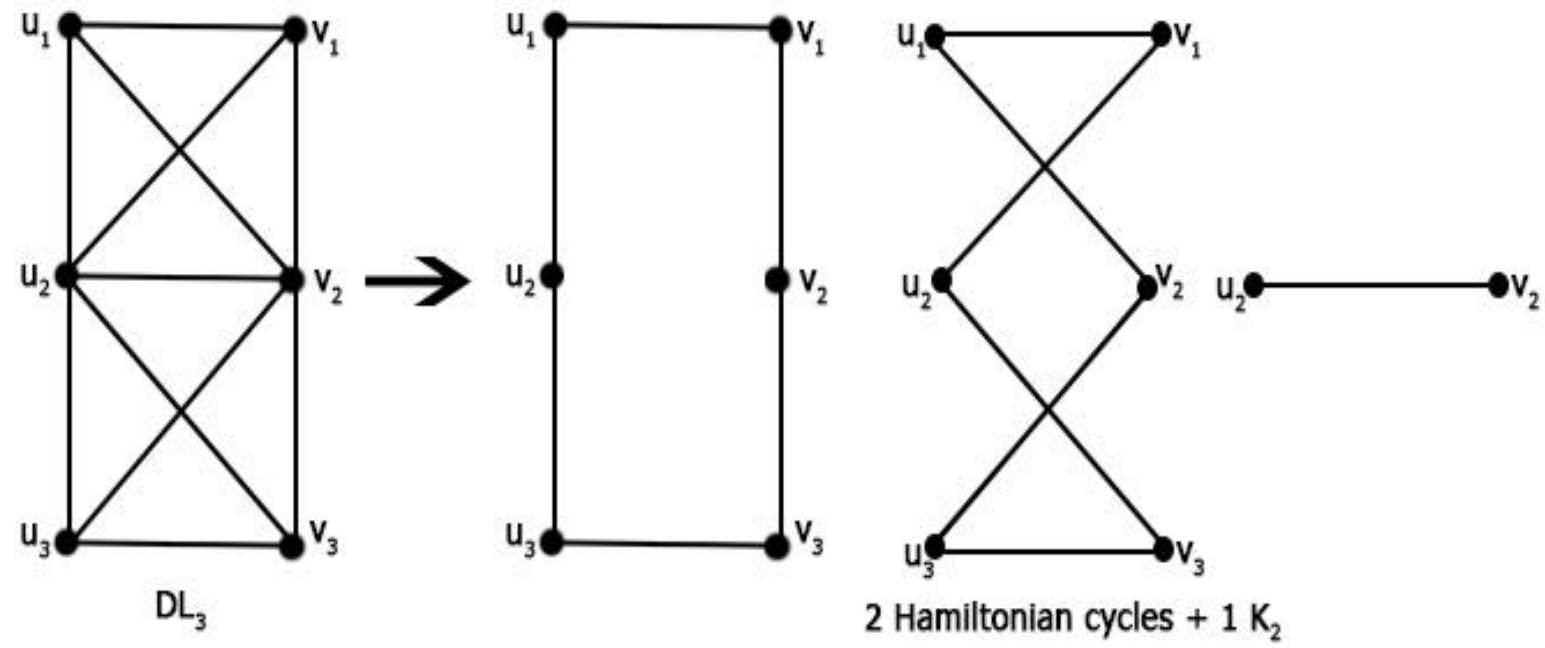

\section{HAMILTONIAN DECOMPOSITION OF CIRCULAR LADDER GRAPH CL}

\section{Theorem: 5.1}

The Circular Ladder graph $\mathrm{CL}_{n}, \mathrm{n} \geq 3$ can be decomposed into $\left\{\begin{array}{c}\mathrm{n} \text { Hamiltonian cyclesif } \mathrm{n} \text { is odd } \\ \mathrm{n}+2 \text { Hamiltonian cyclesif } \mathrm{n} \text { is even }\end{array}\right.$ and the rest of the edges form the 1-factorization.

Proof:

Let $\mathrm{CL}_{\mathrm{n}}, \mathrm{n} \geq 3$ be the Circular Ladder graph with vertex set $\mathrm{V}:\left\{\mathrm{u}_{1}, \mathrm{u}_{2}, \mathrm{u}_{3}, \ldots, \mathrm{u}_{\mathrm{n}}, \mathrm{v}_{1}, \mathrm{v}_{2}, \mathrm{v}_{3}, \ldots, \mathrm{v}_{\mathrm{n}}\right\}$. We consider two cases to prove this theorem.

Case 1: $\mathrm{n}$ is odd

We construct $\mathrm{n}$ paths in the inner cycle

$\mathrm{Q}_{1}: \mathrm{u}_{1} \mathrm{u}_{2} \mathrm{u}_{3} \ldots \mathrm{u}_{\mathrm{n}-2} \mathrm{u}_{\mathrm{n}-1} \mathrm{u}_{\mathrm{n}}$,

$\mathrm{Q}_{2}: \mathrm{u}_{2} \mathrm{u}_{3} \mathrm{u}_{4} \ldots \mathrm{u}_{\mathrm{n}-1} \mathrm{u}_{\mathrm{n}} \mathrm{u}_{1}$,

$Q_{n}: u_{n} u_{1} u_{2} \ldots u_{n-3} u_{n-2} u_{n-1}, n \geq 3$.

Also, we construct $\mathrm{n}$ paths in the outer cycle

$\mathrm{R}_{1}: \mathrm{v}_{1} \mathrm{v}_{2} \mathrm{v}_{3} \ldots \mathrm{v}_{\mathrm{n}-2} \mathrm{v}_{\mathrm{n}-1} \mathrm{v}_{\mathrm{n}}$,

$\mathrm{R}_{2}: \mathrm{v}_{2} \mathrm{v}_{3} \mathrm{v}_{4} \ldots \mathrm{v}_{\mathrm{n}-1} \mathrm{v}_{\mathrm{n}} \mathrm{v}_{1}$,

$\mathrm{R}_{\mathrm{n}}: \mathrm{v}_{\mathrm{n}} \mathrm{v}_{1} \mathrm{v}_{2} \ldots \mathrm{v}_{\mathrm{n}-3} \mathrm{v}_{\mathrm{n}-2} \mathrm{v}_{\mathrm{n}-1}, \mathrm{n} \geq 3$.

Now, we connect the end vertices of each paths $Q_{i}$ and $R_{i}, \quad 1 \leq i \leq n, n \geq 3$, we get $n$ spanning cycles.

Case 2: $\mathrm{n}$ is even 
We construct $n$ paths in the inner cycle

$\mathrm{Q}_{1}: \mathrm{u}_{1} \mathrm{u}_{2} \mathrm{u}_{3} \ldots \mathrm{u}_{\mathrm{n}-2} \mathrm{u}_{\mathrm{n}-1} \mathrm{u}_{\mathrm{n}}$,

$\mathrm{Q}_{2}: \mathrm{u}_{2} \mathrm{u}_{3} \mathrm{u}_{4} \ldots \mathrm{u}_{\mathrm{n}-1} \mathrm{u}_{\mathrm{n}} \mathrm{u}_{1}$,

$Q_{n}: u_{n} u_{1} u_{2} \ldots \quad u_{n-3} u_{n-2} u_{n-1}, n \geq 4$.

Also, we construct $\mathrm{n}$ paths in the outer cycle,

$\mathrm{R}_{1}: \mathrm{v}_{1} \mathrm{v}_{2} \mathrm{v}_{3} \ldots \mathrm{v}_{\mathrm{n}-2} \mathrm{v}_{\mathrm{n}-1} \mathrm{v}_{\mathrm{n}}$,

$\mathrm{R}_{2}: \mathrm{v}_{2} \mathrm{v}_{3} \mathrm{v}_{4} \ldots \mathrm{v}_{\mathrm{n}-1} \mathrm{v}_{\mathrm{n}} \mathrm{v}_{1}$

$\mathrm{R}_{\mathrm{n}}: \mathrm{v}_{\mathrm{n}} \mathrm{v}_{1} \mathrm{v}_{2} \ldots \mathrm{v}_{\mathrm{n}-3} \mathrm{v}_{\mathrm{n}-2} \mathrm{v}_{\mathrm{n}-1}, \mathrm{n} \geq 4$.

Now, we connect the end vertices of each paths $\mathrm{Q}_{\mathrm{i}}$ and $\mathrm{R}_{\mathrm{i}}, 1 \leq \mathrm{i} \leq \mathrm{n}, \mathrm{n} \geq 4$, we get $\mathrm{n}$ spanning cycles. Also, we construct two more spanning cycles, $C_{1}: u_{1} v_{1} v_{2} u_{2} \ldots u_{n-1} v_{n-1} v_{n} u_{n} u_{1}$ and $C_{2}: u_{2} v_{2} v_{3} u_{3} \ldots u_{n} v_{n} v_{1} u_{1} u_{2}$. Therefore, if $n$ is even, we get $n+2$ spanning cycles.

The remaining edges of Circular Ladder graph $\mathrm{CL}_{n}, \mathrm{n} \geq 3$ in both cases form the 1-factorization.

\section{Example: 5.2}
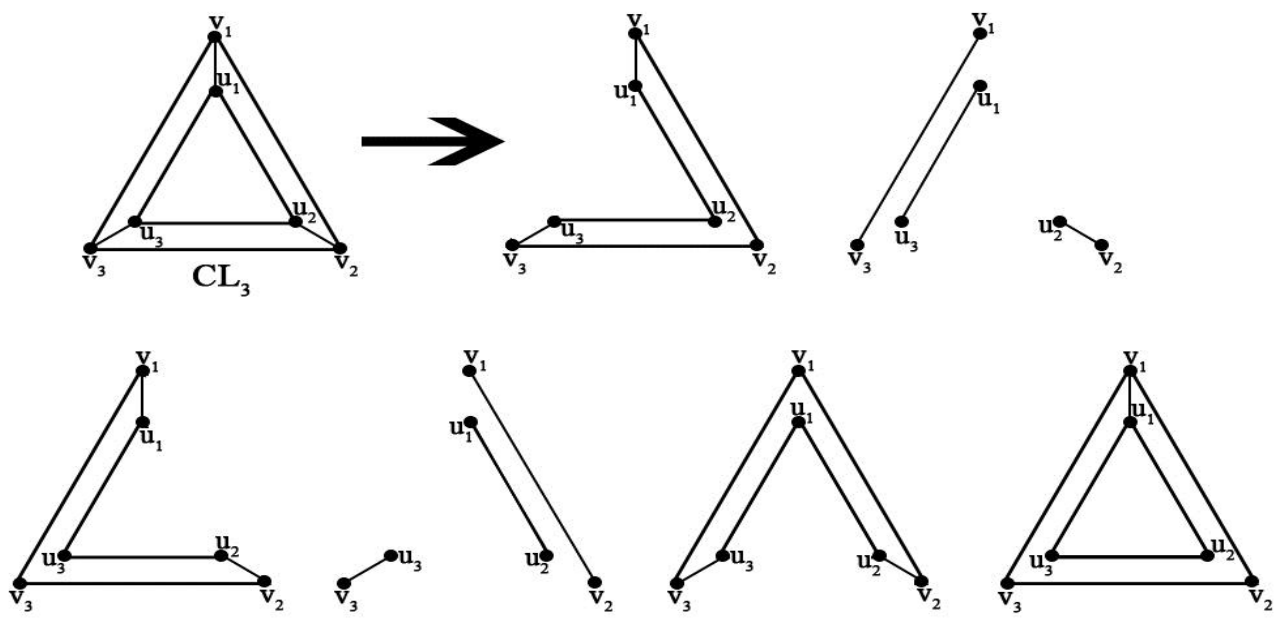

3 Hamiltonian cycles +1 factorization 

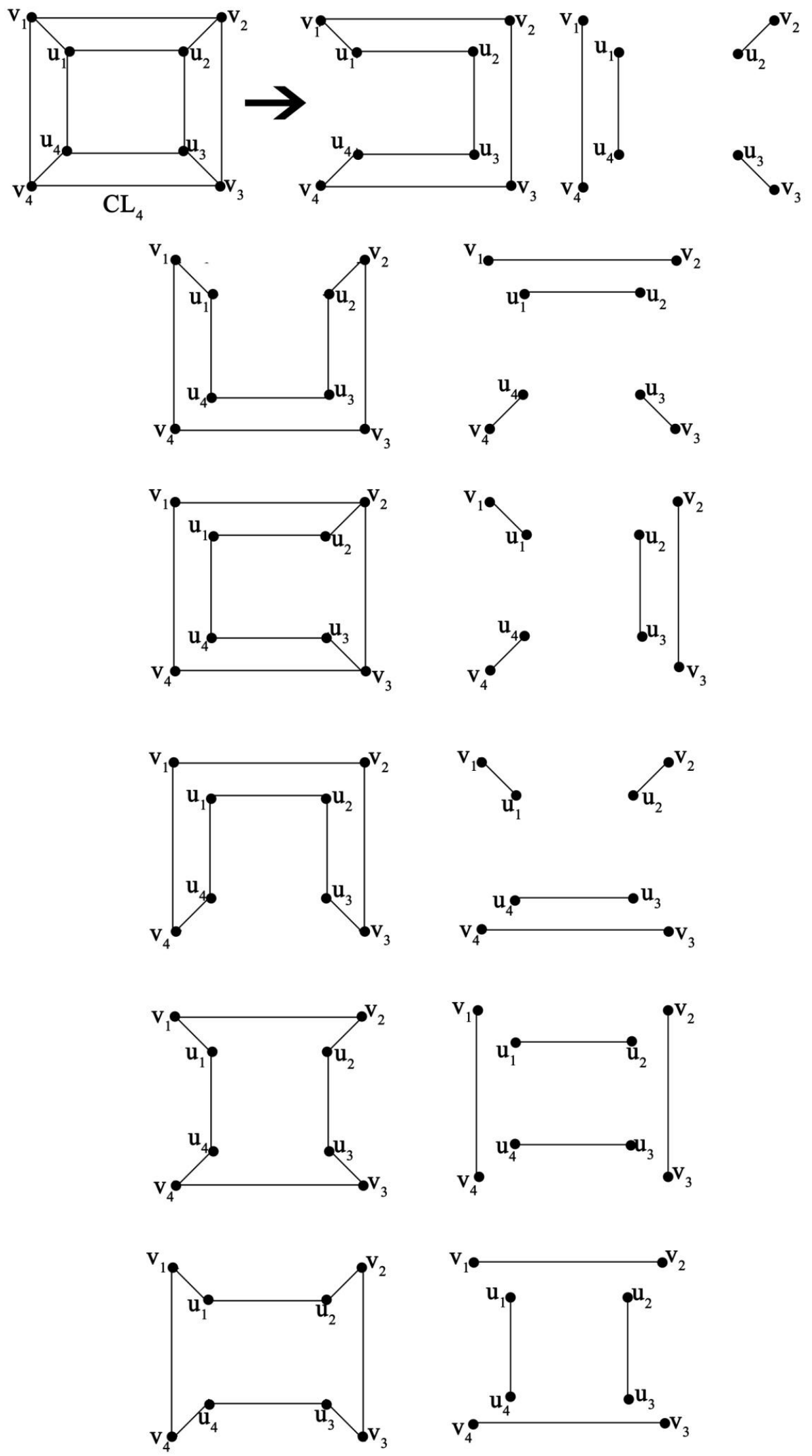

6 Hamiltonian cycles +1 factorization 


\section{CONCLUSION}

We investigate Hamiltonian decomposition of some families of graphs such as ladder, triangular ladder, circular ladder graphs using the concept of Hamiltonian decomposition of graphs. Also, we investigate Hamiltonian decomposition of diagonal ladder graphs using the concept of Hamiltonian decomposition of ladder graphs.

\section{REFERENCES}

[1] Klas Markstrom, "Even cycle decomposition of 4-regular graphs and line graphs".

[2] Murugesan N., Vanadhi T., "Hamiltonian decomposition of complete graphs and complete digraphs", Global Journal of theoretical and applied Mathematics Sciences, Vol 1, No 1, (2011), ISSN 2248-9916.

[3] Bermond J.C., " Hamiltonian Decompositions of Graphs, Directed Graphs and Hypergraphs", Advances in Graph Theory, Annals of Discrete Mathematics 3 (1978) 21-28.

[4] Bondy J.A., Murty U.S.R., "Graph Theory with Applications”, 1976, Elsevier, New York.

[5] Bosak J., "Decompositions of Graphs", 1990, Kluwer Academic Press, Dordrecht.

[6] Seoud. M.A., Shakir M. Salman, “On Difference Cordial Graphs”, Mathematica Aeterna, Vol. 5, 2015, no. 1, 105 - 124.

\section{AUTHORS PROFILE}

Mrs. L.T. Cherin Monish Femila pursued M.Sc and M.Phil from Manonmaniam Sundaranar University, Tirunelveli in 2014 \& 2015. Her area of research is Graph Theory. Her main research work focuses on Decomposition of graphs.

Dr. S. Asha pursued M.Sc., M.Phil. and Ph.D from Manonmaniam Sundaranar University, Tirunelveli in 2004, $2006 \&$ 2012. She is currently working as Assistant Professor in Department of Mathematics from Nesamony Memorial Christian College, Marthandam since 2006. Her area of research is Graph Theory. Her main research work focuses on Decomposition of graphs. She has 13 years of teaching experience and 4 scholars doing Ph.D under her guidance.

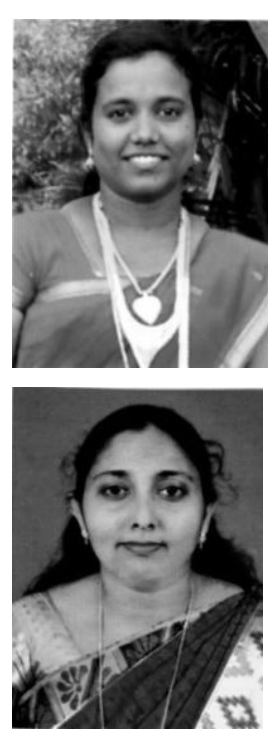

